\title{
Observational study of visual testing efficacy in detecting cannabis usage
}

\author{
Phillip Olla, ${ }^{*}$ Mustafa Abumeeiz,* Lauren Elliott*, Rachel Foote, Mialynn Lee-Daigle, \\ Stephen Bartol*, and Laszlo Erdodi ${ }^{*}$
}

\begin{abstract}
Drug recognition and examination programs are widely used to detect drug impairment in motor vehicle operators. Visual tests are a key assessment in the detection of cannabis-related impairment. Participants were recruited via social media from the medical cannabis community in Southwestern Ontario, Canada. Twenty-two participants completed the full observational trial design. The majority ( $n=13$ or $59.1 \%$ ) were male, with a mean age of 36 years ( $\mathrm{SD}=9.4$; range: $24-59)$. Participants underwent the following protocol: 1) First round of testing (vital signs, bio sample collection, visual tests, subjective data, neurocognitive testing) (Baseline phase); 2) Consumption of cannabis via inhalation; 3) Second round of testing 30 minutes following consumption (THC phase); 4) Additional rounds of testing at 90, 150, and 210 minutes following consumption (Recovery phase). Visual assessment data and vital signs did not follow typical patterns associated with acute cannabis intoxication. With blood THC levels more than double the Canadian legal limit $(5 \mathrm{ng} / \mathrm{mL})$, visual testing results were not diagnostic for cannabis impairment, as participants maintained normal pupil sizes and normal ocular convergence patterns. Visual testing is a key component in standardized examinations used for detecting cannabis-related impairment in Canadian drivers; however, our data indicate that visual testing may not be an effective diagnostic tool for the specific population of medical cannabis users.
\end{abstract}

Key Words Medical cannabis; impaired driving; drug recognition expert.

\section{INTRODUCTION}

Cannabis is the most widely used illicit drug worldwide (Hamilton et al., 2017; Health Canada, 2017). While commonly used for medicinal purposes (for example, it is prescribed by doctors in Canada as per the Cannabis Act) or recreationally in a decriminalized context, there has been recent broader legalization of cannabis for recreational use in Canada and in several American states, such as Montana, Arizona, and New Jersey (Cox, 2018; Health Canada, 2020; National Conference of State Legislatures, 2021). Given the trend towards the legal allowance of cannabis use, the health and safety risks of the substance, especially implications for impaired driving, are a primary concern.

A study by Hartman et al. (2016) finds cannabis to be the most common illicit drug identified in cases of driving under the influence. Cannabis is known to induce cognitive and psychomotor changes that result in impaired driving and, as such, has been linked to acute harm outcomes including increased risk of a motor vehicle accident (Busardò et al., 2017;
Fischer et al., 2018; Hartman et al., 2016). Psychophysical and visual examinations have been previously shown to produce reliable indicators of cannabis intoxication in impaired driving cases (Hartman et al., 2016), but less is known about the effectiveness of standard impairment testing in the specific population of medical cannabis users.

\section{Review of Drug Impairment Examinations}

The Drug Evaluation and Classification (DEC) program is one of the most widely used procedures for assessing drug impairment in drivers and provides a systematic means for law enforcement to find the cause of impairment in drivers suspected of being under the influence of drugs (PorathWaller et al., 2009; Richman, 2017). The DEC program contains a 12-step protocol for law enforcement; police officers can become accredited by the International Association of Chiefs of Police, through the RCMP, as Drug Recognition Experts (DRE) who use the DEC (see Figure 1; Royal Canadian Mounted Police, 2018a). Previous research has found DEC examinations to be up to $95 \%$ accurate in correctly identifying

Correspondence to: Phillip Olla, Audacia Bioscience, Suite 105, 880 North Service Rd, Windsor, ON N8X 3H6, Canada. E-mail: Phillip@audaciabio.com. To cite: Olla, P., Abumeeiz, M., Elliott, L., Foote, R., Lee-Daigle, M., Bartol, S., \& Laszlo, E. (2021). Observational study of visual testing efficacy in detecting cannabis usage. Journal of Community Safety and Well-Being, 6(2), 83-92. https://doi.org/10.35502/jcswb.176

@ Author(s) 2021. Open Access. This work is distributed under the Creative Commons BY-NC-ND license. For commercial re-use, please contact sales@sgpublishing.ca. gg PUBLISHING Published by SG Publishing Inc. CSKA Official publication of the Community Safety Knowledge Alliance. 


\section{Drug Recognition Expert Evaluation}

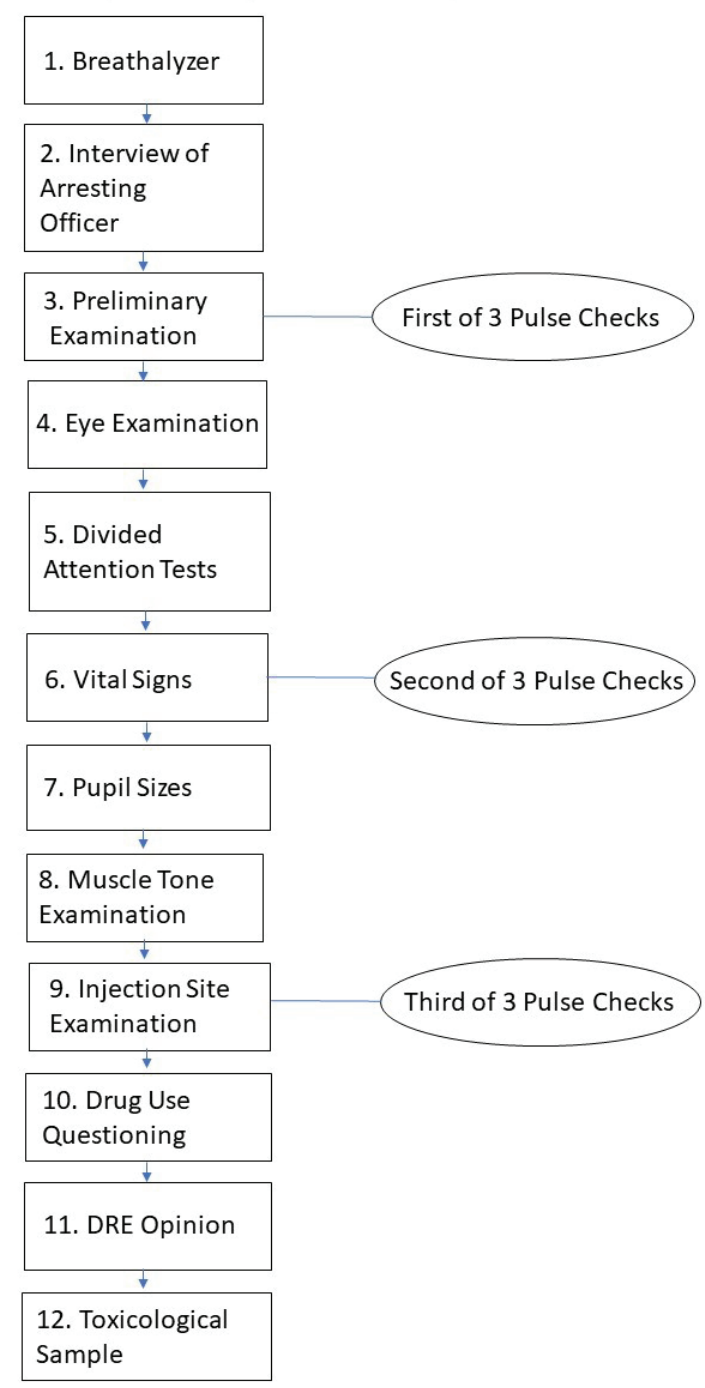

FIGURE $1 \quad$ 12-step drug recognition expert evaluation flow chart (produced with information from Royal Canadian Mountain Police, 20181. DRE $=$ drug recognition expert.

drug impairment in Canada (Beirness et al., 2009; PorathWaller \& Beirness, 2010; Smith et al., 2002).

Standardized field sobriety tests (SFSTs) are widely used by law enforcement officers to detect drug and alcohol impairment in drivers and are included in the DEC. These tests are brief psychophysical tests done roadside (including limited visual exam and walk-and-turn test) that screen for impairment (Government of Canada, 2018). If the driver's blood alcohol content is inconsistent with the arresting officer's observation of impairment via the SFST, the rest of the DEC evaluation can be used to ascertain the nature of the involved substance. This evaluation is completed by DREs in a controlled environment, often at a police department. The DEC includes components such as a pupil examination and an eye examination as well as breath alcohol testing, psychophysical coordination testing, vital sign measurement, muscle tone examination, interviews with the offender and arresting officer, and toxicological examination (Hartman et al., 2016; Porath-Waller et al., 2009; Royal Canadian Mounted Police, 2018a). Based on the combined results of these tests, examiners classify the offending substance into one or multiple of seven categories, one of which is cannabis (International Drug Evaluation and Classification Program, n.d.; Richman, 2017).

\section{Using Visual Assessments to Detect Impairment}

An important aspect of SFSTs and the DEC is visual testing. While visual testing in SFSTs is only comprised of horizontal and vertical gaze-evoked nystagmus (sustained jerking when eyes are deviated to the side; Royal Canadian Mounted Police, 2018a), during the DEC, pupil size changes (pupil size and reaction to varied lighting conditions) and potential lack of convergence (inability to sustain inward turning of eyes) are also examined. In combination with the other parameters of the DEC, visual testing can be used to profile various forms of substance impairment including ethanol, cocaine, and cannabis impairment. Research has identified that during visual testing, ethanol produces horizontal gaze nystagmus, does not cause lack of convergence, and does not cause pupil dilation (Heishman et al., 1996; Romano et al., 2017). In combination with errors in the walk-and-turn test, miscounting in and failure to complete the one-leg-stand test, abnormal muscle tone, and increased pulse readings, ethanol impairment can be identified through the DEC (Heishman et al., 1996). Similarly, cocaine produces pupil dilation, does not cause lack of convergence, and does not cause horizontal gaze nystagmus (Heishman et al., 1996; National Institute on Drug Abuse, 2020). In combination with errors in the walk-and-turn test, decreased errors on the one-leg-stand test, abnormal muscle tone, and increased pulse readings, cocaine impairment can also be identified through the DEC (Heishman et al., 1996).

\section{Using Visual Assessments to Detect Cannabis Impairment}

Previous researchers have established the association of acute cannabis intoxication with pupil dilation (Bramness et al., 2010; Heishman et al., 1996) and lack of convergence (Hartman et al., 2016; Heishman et al., 1996; Yeakel \& Logan, 2013). Additionally, cannabis impairment is associated with normal results for gaze-evoked nystagmus (Hartman et al., 2016; Heishman et al., 1996; Kibby \& Halcomb, 2013; PorathWaller \& Beirness, 2010; Yeakel \& Logan, 2013). This visual assessment, in combination with other DEC measures such as errors on the walk-and-turn test, abnormal muscle tone, decreased errors on the finger-to-nose test, and increased pulse readings (Heishman et al., 1996), provides a profile indicative of individuals under the influence of cannabis. While these are the established expected visual findings for cannabis impairment in the general population, potential differences in visual assessment findings between medical cannabis users and less frequent or recreational users has yet to be investigated.

\section{Examining Medical Cannabis Users}

Medical cannabis users may differ from recreational users in the frequency and chronicity of their cannabis use. Medical cannabis patients could be more likely to partake in daily and 
chronic use due to their medical needs (Hill, 2015). Through this frequent use, individuals can develop a physiological tolerance to the acute effects of the drug (Colizzi \& Bhattacharyya, 2018; Desrosiers et al., 2015; Theunissen et al., 2012). If medicinal cannabis users display less significant signs of impairment due to tolerance, it is possible that standard examinations for the presence of impairment may provide inaccurate results. Medical users may also have cannabinoids lingering in their system due to frequent use and due to various ingestion methods. The RCMP can test either oral fluid, urine, or blood (Royal Canadian Mounted Police, 2018b). THC can be detectable in blood at greater than the legal limit $(2 \mathrm{ng} / \mathrm{mL})$ for up to a week in frequent cannabis users (Peng et al., 2020), which would modulate both their baseline results and their results following subsequent dosages of cannabis. This brings into question whether the eye and pupil examination portions of the DEC, and the rest of the protocol, for that matter, are suitable for use with medical cannabis users. This research seeks to investigate theeffectiveness of visual testing in the detection of cannabis impairment for medical cannabis patients. In particular, it will focus on aspects of eye examinations that are not present in SFSTs and which are important in identifying cannabis impairment specifically (lack of convergence and pupil dilation).

\section{Ethics Approval and Consent to Participate}

This project was approved by the University Research Ethics Board, and ethical guidelines regulating research involving human participants were followed throughout the study. All data collection, storage, and processing were done with the approval of relevant institutional authorities regulating research involving human participants, in compliance with the 1964 Helsinki Declaration and its subsequent amendments or comparable ethical standards. Study participants gave informed consent at every phase of the trial. The datasets used and/or analyzed during the current study are available from the corresponding author on reasonable request.

\section{METHODS}

The aim of this observational study was to investigate the neurocognitive and psychophysiological effects of acute cannabis intoxication in a sample of medical cannabis users. Participants were recruited via social media from the medical cannabis community in Southwestern Ontario, Canada. Three hundred people expressed interest in participating in the study, of which 30 completed a medical interview via a telemedicine service to verify eligibility. Twenty-three participants reported to the study, but one withdrew early due to adverse effects following cannabis consumption. Thus, a total of 22 participants completed the full study design.

The majority of participants $(n=13$ or $59.1 \%)$ were male. Mean age was 36.0 years ( $\mathrm{SD}=9.4$; range: $24-59$ ). The mean level of education was 13.7 years, 12 years being a high school degree ( $\mathrm{SD}=1.7$; range: $10-16)$. To be included in the study, participants were required to be 24 years of age or older and native English speakers, in possession of a medical marijuana license for treatment of a chronic health condition, medically stable, and have peripheral veins suitable for repeated venipuncture. Exclusion criteria were pregnancy and allergy to any cannabinoid or marijuana smoke. The most common reason for participants' medical marijuana prescription was a psychiatric disorder $(n=15$ or $68.2 \%)$, followed by musculoskeletal ( $n=4$ or $18.2 \%$ ), (auto)immune ( $n=2$ or $9.1 \%$ ) and respiratory ( $n=1$ or $4.5 \%$ ) illnesses. Pain management was identified as one of the reasons for which medical marijuana was prescribed in $54 \%$ of the sample $(n=12)$. Average selfreported cannabis consumption was 3.2 grams/day $(\mathrm{SD}=1.5$, range: 1-14) and mean body-mass index was 29.78 (SD = 9.5; range: $17.77-51.62)$. More details about individual participants can be found in Table I.

\section{Procedure}

Participants were observed over six hours on a single day from 8:30 a.m. to 3:00 p.m. Height and weight, vital signs (blood pressure, heart rate, temperature), urine samples, and breath samples were collected by registered nurses, and blood samples (plasma THC levels) were collected by phlebotomists. A medical doctor was available for the duration of the study. Neurocognitive tests were administered by research assistants with extensive training in test administration and scoring who had previous experience administering these measures in research and clinical settings. The research assistants were supervised by a licensed clinical neuropsychologist. These measures, as well as pupil size, ocular convergence, and subjective data were taken at 30,90, 150, and 210 minutes following cannabis consumption. Participants' breath was collected using an exhaled breath collection device called ExaBreath produced by SensAbues AB of Sweden. Samples were analyzed using Liquid ChromatographyTandem Mass Spectrometry at the Hospital for Sick Children, Toronto, Canada. Subjective data included data regarding emotional state and physical symptoms. These included subjective ratings of cannabis "high," feelings of relaxation, happiness, slow reaction, confusion, concentration, energy, depression, anxiety, dizziness, nausea, hunger, pain, and more. Following the baseline measurements, one gram of Cannabis sativa (20\% THC) was consumed by participants via vapes, cannabis cigarettes (joints), and dabs for 10 minutes, followed by subjective level of intoxication reporting on a visual analogue scale. It is important to note that participants were medical cannabis users and had therefore consumed their medical dosages before the study and presented with elevated blood THC levels. Dosage amounts were specific to each participant.

\section{Plasma THC Measurement}

Plasma THC levels were measured at baseline (pre-consumption) and at 30, 90, 150, and 210 minutes post-consumption. Plasma THC measurements were performed by the Analytical Facility for Bioactive Molecules at the Hospital for Sick Children. The measured plasma THC values were then converted to their blood THC equivalents for legal limit comparison, using a plasma-to-blood conversion model commonly employed in the literature (Desrosiers et al., 2014; Huestis et al., 2005).

\section{Visual Testing}

Eye movement and pupil size were observed pre-consumption and at 30, 90, 150, and 210 minutes post-consumption. Visual assessments were completed by a police officer trained in DEC 
TABLE I Research participants' medical cannabis usage profile and eye convergence data prior to consumption

\begin{tabular}{|c|c|c|c|c|c|c|}
\hline Participant & BMI & Age & Usage per Day (g) & Gender & Medical Condition & Lack of Convergence \\
\hline 1 & 28.14 & 32 & 3 & Male & Back pain & $\mathrm{N}$ \\
\hline 2 & 29.18 & 42 & 2 & Female & Back pain & $\mathrm{N}$ \\
\hline 3 & 31.31 & 30 & 3.5 & Male & Chronic pain & $N$ \\
\hline 4 & 19.67 & 44 & 1 & Female & Degenerative disc disorder & $N$ \\
\hline 5 & 26.57 & 33 & 2 & Female & Anxiety & $N$ \\
\hline 6 & 22.36 & 37 & 2.5 & Female & Depression & $Y^{a}$ \\
\hline 7 & 44.26 & 36 & 5 & Male & Anxiety & $N$ \\
\hline 8 & N/A & 25 & 1 & Male & Anxiety & $N$ \\
\hline 9 & 46.68 & 26 & 2 & Female & Immune & $\mathrm{N}$ \\
\hline 10 & 34.26 & 35 & 2 & Female & Knee pain & Y \\
\hline 11 & 22.83 & 37 & 3 & Male & Anxiety & Ya \\
\hline 12 & 17.77 & 27 & 2 & Male & Arthritis & $\mathrm{N}$ \\
\hline 13 & 19.59 & 33 & 2 & Male & Scoliosis & $\mathrm{N}$ \\
\hline 14 & 20.90 & 34 & 2 & Male & Osteoarthritis & $\mathrm{N}$ \\
\hline 15 & 34.08 & 39 & 1.25 & Male & Lower back pain & $N$ \\
\hline 16 & 22.54 & 31 & 3 & Female & Osteoarthritis & $\mathrm{N}$ \\
\hline 17 & 35.62 & 58 & 1.5 & Female & Anxiety & $\mathrm{N}$ \\
\hline 18 & 30.64 & 59 & 14 & Male & Spinal dysraphism & $Y^{a}$ \\
\hline 19 & 29.10 & 32 & 9 & Male & Anxiety & $\mathrm{N}$ \\
\hline 20 & 27.70 & 39 & 3.5 & Female & Sleep apnea & $\mathrm{N}$ \\
\hline 21 & 18.73 & 24 & 3 & Male & Back pain & $Y^{b}$ \\
\hline 22 & 51.62 & 30 & 1.5 & Male & Back pain & $\mathrm{N}$ \\
\hline 23 & 41.53 & 41 & 3 & Male & Chronic pain & $\mathrm{N}$ \\
\hline
\end{tabular}

a $=$ participants with abnormalities in visual assessment (e.g., eyes drifting to one side, focused to left or right); ${ }^{b}=$ participant with a known medical condition affecting the eyes.

$\mathrm{N} / \mathrm{A}$ = data unavailable.

examinations (the police officer being a DRE), thus reflecting the field standard for the detection of drug impairment. Pupil assessment involved measurement of each pupil diameter and reactivity to light in normal room lighting conditions. Ocular convergence tests assess participants' ability to converge their eyes towards a target (Richman, 2017). In this test, the target (a pen) is positioned approximately 12 inches in front of the participant's face, and the participant is instructed to follow the target movement with the eyes only. The target is moved in two large circles and the eyes are watched to ensure proper tracking. After the circles are completed the pen is positioned back to the center and moved toward the bridge of the nose while the eyes are observed for sustained convergence (inward turning of the eyes) for at least two seconds in duration.

\section{RESULTS}

Due to the length of the study procedure, many participants opted to not participate in subsequent measurement periods. The amount of data collected therefore significantly decreased as consumption periods progressed. There were
22 participants for measurement at baseline, 30 minutes post-consumption, and 90 minutes post-consumption. This dropped as low as 13 at 150 minutes post-consumption and 6 at 210 minutes post-consumption for certain measures (such as pupil dilation and convergence). This means a full comparison can be made across the first 3 points of measurement, and the last 2 comparisons should be interpreted with those limitations in mind. At baseline, we found that out of 21 participants, 4 had a blood level below $2 \mathrm{ng} / \mathrm{mL}, 3$ had $2-$ $5 \mathrm{ng} / \mathrm{mL}, 6$ had $5-10 \mathrm{ng} / \mathrm{mL}$, and 8 exceeded $10 \mathrm{ng} / \mathrm{mL}$ (double the legal limit) (see Figure 2 and Table II). At 30 minutes postconsumption, all participants' blood THC concentrations were greater than $10 \mathrm{ng} / \mathrm{mL}$, blood THC levels well over the current Canadian legal limit of $5 \mathrm{ng} / \mathrm{mL}$. This physiological indication of acute intoxication aligned with an average selfreport of subjective "high" of 5.07 on a scale of zero to ten, compared with an average baseline subjective high of 1.33 . Participants' THC levels remained elevated for more than 90 minutes post-consumption, with 18 of 22 participants maintaining THC levels over $5 \mathrm{ng} / \mathrm{mL}$.

Participants' pupil diameters were measured at intervals throughout the study. Reference pupil sizes for non-impaired 


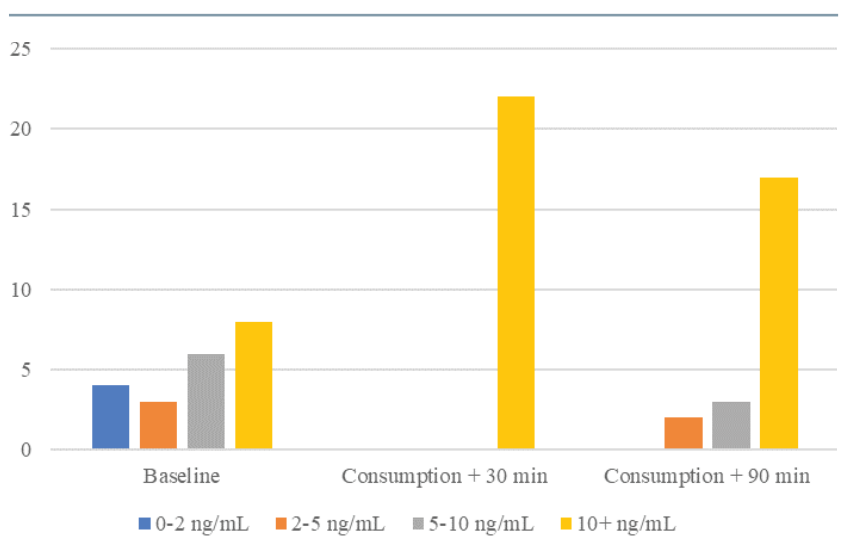

FIGURE 2 Trial blood THC levels in comparison with Canadian per se blood THC limits.

TABLE II Blood THC (ng/mL) across consumption periods

\begin{tabular}{|c|c|c|c|c|}
\hline $\begin{array}{l}\text { Subject } \\
\text { Number }\end{array}$ & Baseline & $\begin{array}{l}\text { Consumption } \\
+30 \text { minutes }\end{array}$ & $\begin{array}{c}\text { Consumption } \\
+90 \\
\text { minutes }\end{array}$ & $\begin{array}{c}\text { Consumption } \\
+150 \\
\text { minutes }\end{array}$ \\
\hline 1 & 15.28 & 29.35 & 32.70 & 19.90 \\
\hline 2 & 1.33 & 15.21 & 5.43 & 4.57 \\
\hline 3 & 1.11 & 42.61 & 14.54 & 9.85 \\
\hline 4 & 6.70 & 11.86 & 3.44 & 2.04 \\
\hline 5 & 5.33 & 33.37 & 15.28 & 8.11 \\
\hline 6 & 6.70 & N/A & N/A & $\mathrm{N} / \mathrm{A}$ \\
\hline 7 & 23.25 & 90.45 & 56.28 & 21.57 \\
\hline 8 & 13.07 & 39.93 & 21.24 & 17.35 \\
\hline 9 & 4.90 & 19.90 & 13.60 & 7.84 \\
\hline 10 & 7.30 & 49.85 & 24.46 & 14.34 \\
\hline 11 & 26.60 & 48.78 & 44.42 & 22.24 \\
\hline 12 & 0.48 & 39.80 & 12.19 & 5.21 \\
\hline 13 & 30.28 & 67.00 & 35.78 & 19.36 \\
\hline 14 & 7.17 & 19.36 & 9.05 & 6.08 \\
\hline 15 & N/A & $\mathrm{N} / \mathrm{A}$ & $N / A$ & $\mathrm{~N} / \mathrm{A}$ \\
\hline 16 & 5.13 & 48.84 & 20.23 & 11.73 \\
\hline 17 & 1.82 & 13.67 & 4.64 & 3.18 \\
\hline 18 & 22.04 & 39.87 & 12.46 & 11.19 \\
\hline 19 & 13.20 & 37.32 & 23.92 & 20.84 \\
\hline 20 & 4.70 & 60.17 & 17.49 & 8.98 \\
\hline 21 & 19.30 & 79.06 & 31.96 & 22.58 \\
\hline 22 & N/A & N/A & N/A & N/A \\
\hline 23 & 2.70 & 21.71 & 7.84 & 5.74 \\
\hline
\end{tabular}

$\mathrm{N} / \mathrm{A}$ = data unavailable.

Note: participant 15 dropped out of the study. individuals in room light range from $2.5 \mathrm{~mm}$ to $5.0 \mathrm{~mm}$, with an average of $4.0 \mathrm{~mm}$ (Drug Recognition Expert Course, 2015; Hartman et al., 2016). In the present study, participants' average pupil sizes ranged from $4 \mathrm{~mm}$ to $4.55 \mathrm{~mm}$ for the right eye and $4 \mathrm{~mm}$ to $4.35 \mathrm{~mm}$ for the left at different measurement points (pre-controlled consumption and post-consumption) (see Table III and Figure 3). There was no spike in pupil size associated with blood THC levels. Throughout the study, pupil sizes remained within what is considered a normal range for non-impaired individuals, and thus were not indicative of impairment from cannabis (Bramness et al., 2010; Heishman et al., 1996).

Eye convergence tests were coded as either "convergence" or "lack of convergence." It is important to note that the majority of participants who opted out of the procedure for the last two measurements were those whose eyes demonstrated

TABLE III Participants' pupil sizes for both left and right eye $(\mathrm{mm})$ across the first three measurement periods

\begin{tabular}{|c|c|c|c|c|}
\hline $\begin{array}{l}\text { Subject } \\
\text { Number }\end{array}$ & $\begin{array}{c}\text { Baseline } \\
\text { L/R } \\
\text { (mm) }\end{array}$ & $\begin{array}{c}\text { Consumption } \\
+30 \text { minutes } \\
\text { L/R } \\
(\mathrm{mm})\end{array}$ & $\begin{array}{c}\text { Consumption } \\
+90 \text { minutes } \\
\text { L/R } \\
(\mathrm{mm})\end{array}$ & $\begin{array}{c}\text { Consumption } \\
+150 \text { minutes } \\
\text { L/R } \\
\text { (mm) }\end{array}$ \\
\hline 1 & $4.2 / 5.9$ & $3 / 3$ & $3 / 3$ & N/A \\
\hline 2 & $4 / 4$ & $4 / 4$ & $4 / 4$ & N/A \\
\hline 3 & $5.3 / 5.3$ & $5 / 5$ & $5 / 5$ & $5.3 / 5$ \\
\hline 4 & $4 / 4$ & $4 / 4$ & $4 / 4$ & N/A \\
\hline 5 & $4 / 4$ & $3 / 3$ & $3 / 3$ & $3 / 3$ \\
\hline 6 & $4.3 / 4.3$ & $4 / 4.2$ & $4 / 4.2$ & $4 / 4.2$ \\
\hline 7 & $6.4 / 6.4$ & $5 / 5$ & $5.3 / 5.3$ & $5.3 / 5.3$ \\
\hline 8 & $5.3 / 5.3$ & $5 / 5$ & $5.3 / 5.3$ & $5.3 / 5.3$ \\
\hline 9 & $4.2 / 4.2$ & $4 / 4$ & $4 / 4$ & $4 / 4$ \\
\hline 10 & $4.2 / 4.2$ & $4.2 / 4.2$ & $4.2 / 4.2$ & $4.2 / 4.2$ \\
\hline 11 & $3 / 4$ & $3 / 3$ & $3 / 3$ & $3 / 3$ \\
\hline 12 & $4.2 / 4.2$ & $3 / 3$ & $3 / 3$ & $3 / 3$ \\
\hline 13 & $5.3 / 5.3$ & $5.3 / 5.3$ & $5.3 / 5.3$ & $\mathrm{~N} / \mathrm{A}$ \\
\hline 14 & $4.2 / 4.2$ & $4.2 / 4$ & $4 / 4$ & N/A \\
\hline 15 & $4.2 / 4.2$ & N/A & N/A & N/A \\
\hline 16 & $4 / 5$ & $4 / 5$ & $4 / 4$ & $4 / 4$ \\
\hline 17 & $4 / 4.3$ & $4 / 4$ & $4.2 / 4$ & $4 / 4$ \\
\hline 18 & $5.4 / 6.5$ & $4 / 5$ & $5 / 4$ & $4 / 5$ \\
\hline 19 & $5.3 / 5.3$ & $5.3 / 5.3$ & $5.3 / 5.3$ & $\mathrm{~N} / \mathrm{A}$ \\
\hline 20 & $2 / 2$ & $2 / 2$ & $2 / 2$ & $\mathrm{~N} / \mathrm{A}$ \\
\hline 21 & $5.3 / 5.3$ & $5 / 5$ & $5 / 5$ & $5 / 5$ \\
\hline 22 & $4 / 4$ & $4.2 / 4.2$ & $4 / 4$ & N/A \\
\hline 23 & $3 / 3$ & $3 / 3$ & $3 / 3$ & $\mathrm{~N} / \mathrm{A}$ \\
\hline Average & $4.35 / 4.55$ & $4 / 4.1$ & $4.1 / 4$ & $4.2 / 4.2$ \\
\hline
\end{tabular}

$\mathrm{N} / \mathrm{A}=$ data unavailable.

Note: participant 15 dropped out of the study. 


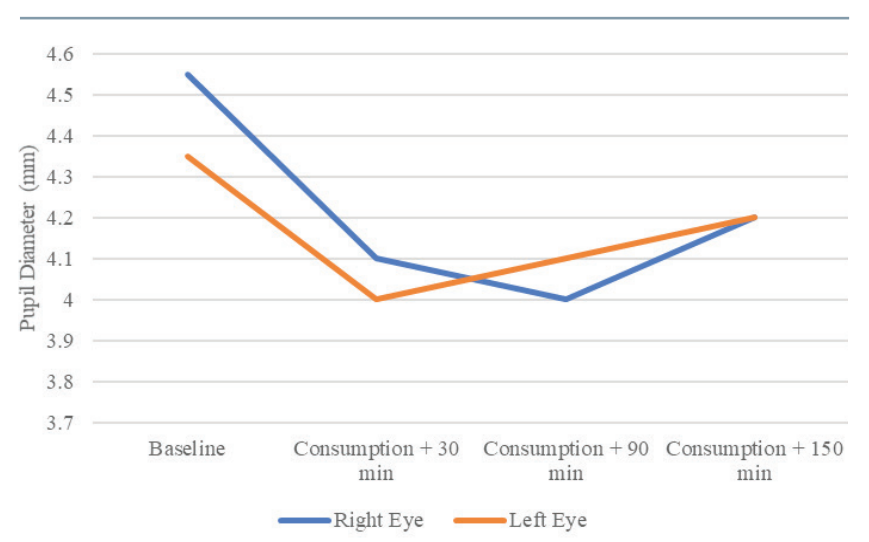

FIGURE 3 Mean pupil sizes for left and right eyes, measured at baseline and at intervals post-cannabis consumption.

normal convergence. Regardless, the majority of participants displayed convergence at each measurement point in the trial, a finding inconsistent with typical signs of cannabis impairment (see Figure 4; Hartman et al., 2016; Heishman et al., 1996; Yeakel \& Logan, 2013). Prior to procedural consumption, 19 of 22 participants displayed convergence. At 30 and 90 minutes post-consumption, 20 and 18 participants displayed convergence, respectively. The results for convergence testing show peculiar variance (see Table IV). Participants 10 and 11 showed convergence patterns expected for acute cannabis intoxication (initial normal convergence, with lack of convergence developing after consumption). However, participants 18 and 21 were unsuccessful in convergence at pre-controlled consumption, and were then able to converge the eyes 30 minutes post-consumption. At the same time, participant 6 displayed lack of convergence at every testing point. During each point of testing, the participants were well above the legal limit for blood THC levels. Overall, participants displayed normal convergence of the eyes at pre-controlled consumption and post-consumption, while simultaneously having blood THC levels significantly higher than the legal limit.

During the examination, vital signs were also observed pre-consumption and at 30,90, 150, and 210 minutes postconsumption. The general trend in our findings is a $12.6 \%$ increase in heart rate 30 minutes following cannabis consumption, with a gradual decrease returning to baseline by 90 minutes for many and by 150 minutes for most (see Figure 5). Paradoxically, however, some participants showed the opposite trend: an initially elevated heart rate with a decrease following cannabis consumption, and a gradual increase in the following hours post-consumption (see Table V, participants $5,8,13$, and 21). While certain gaps and obscure trends made it difficult to interpret subjective data outside basic measurements such as perceived "high," anxiety proved to be a somewhat consistent measure. Measures of anxiety may serve to reflect changes in heart rate; as per Table VI, on average, there was an increase in subjective anxiety that aligned with the spike in heart rate. Average subjective reports of anxiety rose from 2.46 at baseline to 3.05 by 90 minutes postconsumption, while average heart rate rose from 84 beats per minute (bpm) at baseline to 100 at 30 minutes postconsumption and $99 \mathrm{bpm}$ at 90 minutes post-consumption (see Tables V and VI). Both these measures fell by 150 minutes

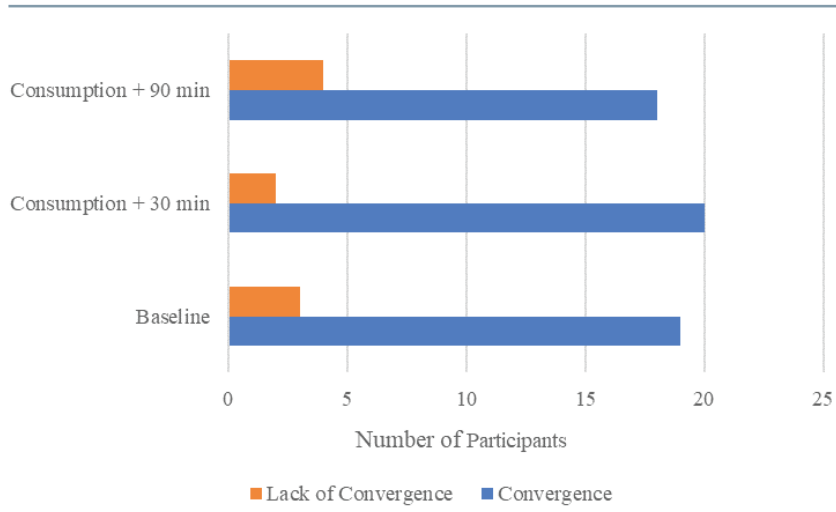

FIGURE 4 Convergence vs lack of convergence at multiple time points in the trial.

TABLE IV Trends for convergence or lack of convergence in participants

\begin{tabular}{|c|c|c|c|}
\hline Subject Number & Baseline & $\begin{array}{l}\text { Consumption } \\
+30 \text { minutes }\end{array}$ & $\begin{array}{l}\text { Consumption } \\
+90 \text { minutes }\end{array}$ \\
\hline 1 & Convergence & Convergence & Convergence \\
\hline 2 & Convergence & Convergence & Convergence \\
\hline 3 & Convergence & Convergence & Convergence \\
\hline 4 & Convergence & Convergence & Convergence \\
\hline 5 & Convergence & Convergence & Convergence \\
\hline 6 & $\begin{array}{c}\text { No } \\
\text { convergence }\end{array}$ & $\begin{array}{c}\text { No } \\
\text { convergence }\end{array}$ & $\begin{array}{c}\text { No } \\
\text { convergence }\end{array}$ \\
\hline 7 & Convergence & Convergence & Convergence \\
\hline 8 & Convergence & Convergence & Convergence \\
\hline 9 & Convergence & Convergence & Convergence \\
\hline 10 & Convergence & Convergence & $\begin{array}{c}\text { No } \\
\text { convergence }\end{array}$ \\
\hline 11 & Convergence & $\begin{array}{c}\text { No } \\
\text { convergence }\end{array}$ & $\begin{array}{c}\text { No } \\
\text { convergence }\end{array}$ \\
\hline 12 & Convergence & Convergence & Convergence \\
\hline 13 & Convergence & Convergence & Convergence \\
\hline 14 & Convergence & Convergence & Convergence \\
\hline 15 & Convergence & $\mathrm{N} / \mathrm{A}$ & $\mathrm{N} / \mathrm{A}$ \\
\hline 16 & Convergence & Convergence & Convergence \\
\hline 17 & Convergence & Convergence & Convergence \\
\hline 18 & $\begin{array}{c}\text { No } \\
\text { convergence }\end{array}$ & Convergence & $\begin{array}{c}\text { No } \\
\text { convergence }\end{array}$ \\
\hline 19 & Convergence & Convergence & Convergence \\
\hline 20 & Convergence & Convergence & Convergence \\
\hline 21 & $\begin{array}{c}\text { No } \\
\text { convergence }\end{array}$ & Convergence & Convergence \\
\hline 22 & Convergence & Convergence & Convergence \\
\hline 23 & Convergence & Convergence & Convergence \\
\hline $\begin{array}{l}\text { Percent of } \\
\text { Participants with } \\
\text { Convergence }\end{array}$ & 87 & 91 & 82 \\
\hline
\end{tabular}

$\mathrm{N} / \mathrm{A}=$ data unavailable.

Note: participant 15 dropped out of the study. 


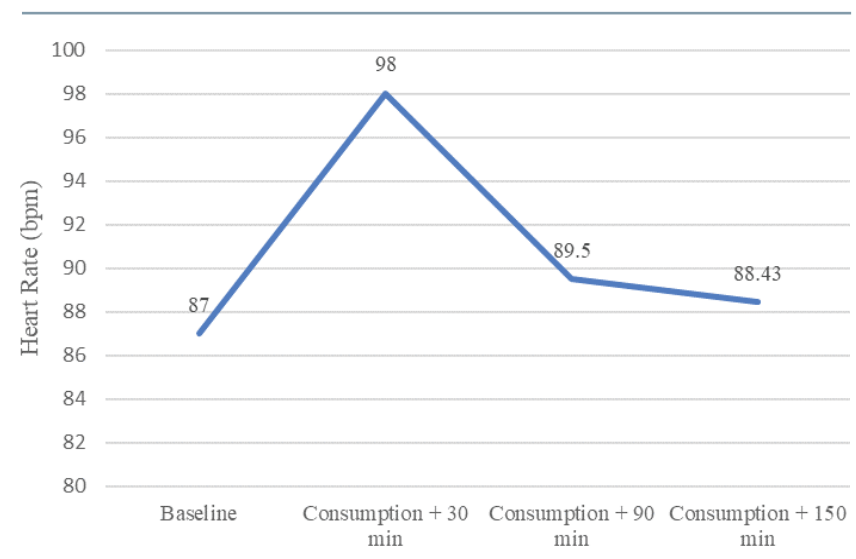

FIGURE 5 Average heart rate of participants throughout the trial.

post-consumption, to 1.50 for anxiety and $77 \mathrm{bpm}$ for heart rate. While this pattern did not hold true for all participants (for example, participants 2 and 6; see Table V and VI), the general trend remains apparent.

\section{DISCUSSION}

This study examined the effect of cannabis on a sample of medical cannabis patients (defined in Methods). Given participants' status as medical cannabis patients, they largely presented with elevated blood THC levels at the beginning of the study. Contrary to previous research on the detection of cannabis impairment, visual assessment data did not follow ty pical patterns associated with acute cannabis ingestion. During the peak THC ingestion phase, $100 \%$ of study participants had blood THC levels over the legal limit but maintained normal visual testing results. According to the DEC and other literature, the expected finding in the context of cannabis intoxication is possible pupil dilation (change greater than $4.0 \mathrm{~mm}$; may be present or absent) and the inability of the eyes to converge (Bramness et al., 2010; Hartman et al., 2016; Richman, 2017; Yeakel \& Logan, 2013). However, in this study, there were no observed changes to pupil size, and minimal changes to convergence during the first two post-consumption measurements. Findings also demonstrated inconclusive results for participants' heart rate post-consumption. In the field, these differences in visual

TABLE V Heart rate before and after consumption of one gram of Cannabis sativa $(20 \% \mathrm{THC})$

\begin{tabular}{|c|c|c|c|c|c|}
\hline $\begin{array}{l}\text { Subject } \\
\text { Number }\end{array}$ & $\begin{array}{l}\text { Baseline blood THC } \\
\text { (ng } / \mathrm{mL})\end{array}$ & $\begin{array}{l}\text { Pre-controlled Consumption } \\
\text { (bpm) }\end{array}$ & 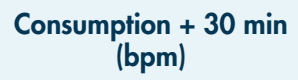 & $\begin{array}{c}\text { Consumption }+90 \text { min } \\
\text { (bpm) }\end{array}$ & $\begin{array}{c}\text { Consumption }+150 \text { min } \\
(\text { bpm) }\end{array}$ \\
\hline 1 & 15.28 & 94 & 105 & 108 & 94 \\
\hline 2 & 1.33 & 89 & 113 & 93 & 98 \\
\hline 3 & 1.11 & 101 & 120 & 103 & 107 \\
\hline 4 & 6.70 & 77 & 93 & 72 & 78 \\
\hline 5 & 5.33 & 67 & 64 & 62 & 69 \\
\hline 6 & 6.70 & 104 & 93 & 94 & 77 \\
\hline 7 & 23.25 & 113 & 114 & 105 & 94 \\
\hline 8 & 13.07 & 86 & 76 & 76 & 82 \\
\hline 9 & 4.90 & 76 & 103 & 101 & 77 \\
\hline 10 & 7.30 & 74 & 105 & 92 & 74 \\
\hline 11 & 26.60 & 80 & 85 & 76 & 71 \\
\hline 12 & 0.48 & 87 & 151 & 125 & 120 \\
\hline 13 & 30.28 & 89 & 84 & 78 & 85 \\
\hline 14 & 7.17 & 79 & 74 & 72 & 72 \\
\hline 15 & $\mathrm{~N} / \mathrm{A}$ & $\mathrm{N} / \mathrm{A}$ & $\mathrm{N} / \mathrm{A}$ & $\mathrm{N} / \mathrm{A}$ & 157 \\
\hline 16 & 5.13 & 82 & 114 & 85 & 98 \\
\hline 17 & 1.82 & 86 & 108 & 100 & 95 \\
\hline 18 & 22.04 & 72 & 90 & 103 & 92 \\
\hline 19 & 13.20 & 87 & 89 & 79 & 76 \\
\hline 20 & 4.70 & 69 & 70 & 70 & 67 \\
\hline 21 & 19.30 & 92 & 61 & 64 & 70 \\
\hline 22 & Data unavailable & 126 & 144 & 112 & 104 \\
\hline 23 & 2.70 & 84 & 100 & 99 & 77 \\
\hline
\end{tabular}

$\mathrm{bpm}=$ beats per minute, $\mathrm{N} / \mathrm{A}=$ data unavailable.

Note: participant 15 dropped out of the study. 
TABLE VI Subjective reports of anxiety on a scale of 1 to 10 across three time periods

\begin{tabular}{|c|c|c|c|}
\hline $\begin{array}{l}\text { Subject } \\
\text { Number }\end{array}$ & Pre-consumption & $\begin{array}{c}90 \text { minutes } \\
\text { post-consumption }\end{array}$ & $\begin{array}{c}150 \text { minutes } \\
\text { post-consumption }\end{array}$ \\
\hline 1 & N/A & 0 & 0.5 \\
\hline 2 & 6 & 3.5 & N/A \\
\hline 3 & N/A & 6 & N/A \\
\hline 4 & $\mathrm{~N} / \mathrm{A}$ & 0.5 & 0.5 \\
\hline 5 & 5 & 4 & 1 \\
\hline 6 & 0 & 8 & 6 \\
\hline 7 & 0 & 0 & 0 \\
\hline 8 & 6 & 3 & 0 \\
\hline 9 & N/A & 0 & 0 \\
\hline 10 & N/A & 5 & 5 \\
\hline 11 & 0 & 0 & 0 \\
\hline 12 & 0 & 0 & 0 \\
\hline 13 & N/A & 3 & 0 \\
\hline 14 & 10 & 0 & 4 \\
\hline 15 & N/A & N/A & N/A \\
\hline 16 & N/A & 5 & 1 \\
\hline 17 & 0 & 10 & 1 \\
\hline 18 & N/A & 7 & 1 \\
\hline 19 & N/A & 1 & 0 \\
\hline 20 & 0 & 0 & 6 \\
\hline 21 & 1 & 2 & 1 \\
\hline 22 & 4 & 8 & 3 \\
\hline 23 & 0 & 1 & 0 \\
\hline Average & 2.46 & 3.05 & 1.50 \\
\hline
\end{tabular}

$\mathrm{N} / \mathrm{A}=$ data unavailable.

Note: participant 15 dropped out of the study.

test results and vital sign inconsistencies may complicate the ability of law enforcement officers to accurately detect cannabis-related impairment in this population.

\section{Lack of Convergence}

The fourth step of a 12-step DEC examination tests the ability of the eyes to converge, which is not present in SFSTs and is typically impaired in the context of cannabis intoxication. Five participants were unable to converge their eyes at one or more points during the trial, three of whom demonstrated this inability at the experimental baseline measurement (prior to in-experiment consumption of cannabis). Of note is that each of the participants who was unable to converge their eyes had an observed ocular abnormality such as involuntary nystagmus or eyes drifting to the right or left. This may have affected their performance on these visual tests. Furthermore, it is known that approximately $7 \%$ of the general population displays an inability to properly converge their eyes at the experimental baseline (Richman, 2017). Overall, the majority of participants maintained the ability to converge their eyes at the experimental baseline, through the acute phase of cannabis ingestion, and through the recovery period. For the few participants whose eyes did not converge, there were known medical factors potentially influencing their visual testing results.

Of additional note is the reduced compliance with visual testing protocols later in the study, which limited data collection for convergence after the 90-minute mark. As per the study protocol, research participants were required to consent to each round of testing. After the initial rounds of testing, some participants who had no impairments in convergence declined to continue with further convergence testing. This resulted in incompleteness of the dataset for these later points in the trial. However, from the perspective of cannabis intoxication, it is unlikely that the ability to converge would be affected beyond 90 minutes post-consumption when it was not affected during the acute phase ( 30 minutes postconsumption). From the data collected, it remains clear that this sample of medical cannabis users did not produce visual testing results typically indicative of cannabis impairment on DEC examinations.

\section{Heart Rate Measurement}

During a DEC examination, the arresting officer measures the driver's heart rate three separate times during the evaluation. Typically, the aim is to identify an increase in heart rate. In this study, the physiological effects of participants' various medical conditions, in combination with the modulating effects of cannabis, may be confounding factors in the interpretation of heart rate measurements. It should be reiterated that subjective anxiety reports (see Table VI) reflect the increases in heart rate post-consumption (30 minutes to 90 minutes, and 90 minutes to 150 minutes), which may provide an explanation for this phenomenon. Since arresting officers will not have baseline (pre-cannabis consumption) heart rate measurements for their suspects, their findings at the road side may only amount to minor decreasing or increasing trends over the three pulse checks. Furthermore, multiple factors will conceivably influence the heart rate of an impaired driving suspect: beyond the true physiological effects of the impairing substance, there will be sympathetic nervous arousal associated with interaction with law enforcement. Overall, the inconsistency in findings makes it challenging to extract valuable conclusions applicable to the context of roadside examination. However, a $12.6 \%$ increase 30 minutes post-consumption was recorded, followed by a return to baseline. This spike may reflect what was expected of the DEC examination following cannabis ingestion (Heishman et al., 1996). The speed of the recovery may also be influenced by the tolerance effects demonstrated by medical users (Bosker et al., 2012; Colizzi \& Bhattacharyya, 2018). Further research in this area would be useful, as there are many factors which may influence vitals when consumption of cannabis occurs.

\section{Tolerance to the Effects of Cannabis}

As medical cannabis patients, participants in this study were frequent users of cannabis, with variable prescribed dosages (see Table I). Participants' generally elevated baseline THC measurements are indicators of recent or chronic use of cannabis. Participants' average subjective feeling of intoxication 
30 minutes after consumption was only 5.07 out of 10 , which is some indication of a level of tolerance. While subjective reports may contain inconsistencies, they prove useful in establishing general trends. Additionally, medical cannabis users may be more able to describe experienced effects. Previous research has shown that medical cannabis users can develop a tolerance to cannabis and subsequently experience reduced acute effects compared with recreational users or otherwise nondaily users (Bosker et al., 2012; Colizzi \& Bhattacharyya, 2018). In this study, the overall normal visual testing results may be related to participants' tolerance to cannabis. However, other researchers have found few differences in cannabis-related neurocognitive impairment among frequent versus infrequent cannabis users, so the connection remains unclear (Ramaekers et al., 2016). Furthermore, as was mentioned, most participants had above a 0 baseline of blood THC, with a large portion having above the legal limit. This pre-existing THC might have affected subsequent results, both at baseline and post-consumption. Ideally, participants would have come in without THC in the blood, but as they were a population of medical cannabis users, that would have required days of abstinence from their medication, which would have been unethical.

\section{Medical Cannabis and Driving}

In Canada, there are currently two levels of cannabis-related impairment. The greatest punishment incurred goes to those found driving with a THC level of 5 ng or more, resulting in a fine and/or variable prison term, while driving with a blood THC level between 2 and $5 \mathrm{ng}$ is a less serious offence (Government of Canada, 2019). Due to the chronic and frequent nature of their cannabis use, medical cannabis patients could have elevated levels of blood THC. As shown in this study, these patients may not display the expected indicators of impairment. Visual testing is a core component of DEC assessments, and typically produces results that may indicate cannabis use. Our data highlight that even when over the legal THC limit, medical cannabis patients are likely to maintain normal pupil sizes, retain the ability to converge the eyes, and may not have expected heart rate patterns following consumption. This brings into question how policy should change given this information. If the potential tolerance effects of medical users and pre-existing THC in their blood may confound results, the DEC should re-evaluate its methods. One option would be use different methods for a driver with a medical cannabis card, but this would not reach frequent recreational users of cannabis who may demonstrate a similar profile to medical users. Tests that accurately and consistently measure impairment regardless of potential tolerance and pre-existing cannabis metabolites should be implemented.

\section{Limitations and Future Research}

This study involved a relatively small sample size of 22 participants. The feasibility of a larger cohort was affected by the logistical complexity of the study design. As discussed above, there was reduced compliance with the visual testing protocols later in the trial, which resulted in incompleteness of the dataset at these points. Additionally, overall difficulties with recruitment at the time limited the potential size and diversity of the participant pool. Had the study been performed more recently, after the legalization of recreational cannabis in Canada, the medical cannabis participant pool could have increased. Furthermore, recreational cannabis users could have been included in the study design, more controllable methods of cannabis ingestion could have been implemented (such as edibles), and groups with little to no cannabis experience could have been included to deepen potential interpretations about tolerance, DEC testing, and visual testing. Future research could explore the link between tolerance, impairment, and visual testing more rigorously by including these additions to improve the potential conclusions drawn. While research has been done on the DEC protocol and other drugs, similar questions related to frequent usage and driving should be asked for these drugs (Heishman et al., 1996; National Institute on Drug Abuse, 2020). All of this research should feed back into the readjustment of DEC policy so that reports of impaired driving can be more accurate, and justice dispersed equitably and according to the best available research.

\section{CONCLUSION}

Visual assessments are important for detecting cannabis impairment during DEC examinations. However, the present data suggest that visual test results are not diagnostic for cannabis impairment in medical cannabis users, despite significantly elevated levels of blood THC. This may present a problem for law enforcement officers in their goal of accurately detecting cannabis-related impairment in this population. The DEC examination has been in use for over 30 years. However, with current trends toward the broad legalization of cannabis, it is timely and necessary to conduct further research in this area and establish appropriate testing for impairment with safety considerations. This research should be communicated with organizations conducting DEC training to establish effective standardization of the test.

\section{ACKNOWLEDGEMENTS}

This project received financial support from the Ontario Center for Excellence. The authors wish to thank Cannascribe for conducting the medical recruitment interviews and the biological Analytical Facility for Bioactive Molecules, The Hospital for Sick Children, Toronto, Canada, for assistance with the measurement of the blood and breath bio samples.

\section{CONFLICT OF INTEREST DISCLOSURES}

The authors have no conflicts of interest to declare.

\section{AUTHOR AFFILIATIONS}

*Audacia Bioscience, Windsor, ON, Canada; ${ }^{\star}$ Department of Psychology, University of Windsor, Windsor, Ontario, Canada.

\footnotetext{
REFERENCES

Beirness, D. J., Beasley, E., \& Lecavalier, J. (2009). The accuracy of evaluations by Drug Recognition Experts in Canada. Canadian Society of Forensic Science Journal, 42(1), 75-79. https://doi.org/10.108 O/00085030.2009.10757598

Bosker, W. M., Kuypers, K. P. C., Theunissen, E. L., Surinx, A., Blankespoor, R. J., Skopp, G., Jeffery, W. K., Walls, H. C., van Leeuwen, C. J., \& Ramaekers, J. G. (2012). Medicinal delta(9)-tetrahydrocannabinol (dronabinol) impairs on-the-road driving performance of occasional and heavy cannabis users but is not detected in Standard Field Sobriety Tests. Addiction, 1071101, 1837-1844. https://doi. org/10.1111/ן.1360-0443.2012.03928.x
} 
Bramness, J. G., Khiabani, H. Z., \& Mørland, J. (2010). Impairment due to cannabis and ethanol: Clinical signs and additive effects. Addiction, 105(6), 1080-1087. https://doi.org/10.1111/i.1360 0443.2010.02911.x

Busardò, F. P., Pellegrini, M., Klein, J., \& di Luca, N.M. (2017). Neurocognitive correlates in driving und $455 \mathrm{er}$ the influence of cannabis. CNS and Neurological Disorders-Drug Targets, 16151, 534-540. https://doi.org/10.2174/1871527316666170424115455

Colizzi, M., \& Bhattacharyya, S. (2018). Cannabis use and the development of tolerance: A systematic review of human evidence. Neuroscience and Biobehavioural Reviews, 93, 1-25. https://doi. org/10.1016/i.neubiorev.2018.07.014

Cox, C. (2018). The Canadian Cannabis Act legalizes and regulates recreational cannabis use in 2018. Health Policy, 122(3), 205-209. https://doi.org/10.1016/i.healthpol.2018.01.009

Desrosiers, N. A., Himes, S. K., Scheidweiler, K. B., Concheiro-Guisan, M., Gorelick, D. A., \& Huestis, M. A. (2014). Phase I and II cannabinoid disposition in blood and plasma of occasional and frequent smokers following controlled smoked cannabis. Clinical Chemistry, 60(4), 631-643. https://doi.org/10.1373/clinchem.2013.216507

Desrosiers, N. A., Ramaekers, J. G., Chauchard, E., Gorelick, D. A., \& Huestis, M. A. (2015). Smoked cannabis' psychomotor and neurocognitive effects in occasional and frequent smokers. Journal of Analytical Toxicology, 39(4), 251-261. https://doi.org/10.1093/jat/bkv012

Drug Recognition Expert Course (2015). www.wsp.wa.gov/breathtest/ docs/dre/manuals/7day/2015/student_7day_oct2015.pdf

Fischer, B., Russell, C., Rehm, J., \& Leece, P. (2018). Assessing the public health impact of cannabis legalization in Canada: Core outcome indicators toward an 'index' for monitoring and evaluation. Journal of Public Health, 41(2), 1-10. https://doi.org/10.1093/pubmed/fdy090

Government of Canada. (2018). Legislative background: Reforms to the transportation provisions of the Criminal Code Bill C-46. www.justice. gc.ca/eng/ci-jp/sidl-rlcfa/c46/p2.html

Government of Canada. (2019). Impaired driving laws. www.justice. gc.ca/eng/ci-ip/sidl-rlcfa/index.html

Hamilton, H. A., Brands, B., lalomiteanu, A. R., \& Mann, R. E. (2017). Therapeutic use of cannabis: Prevalence and characteristics among adults in Ontario, Canada. Canadian Journal of Public Health, 108(3), e282-e287. https://doi.org/10.17269/CJPH.108.6130

Hartman, R. L., Richman, J. E., Hayes, C. E., \& Huestis, M. A. (2016). Drug Recognition Expert (DRE) examination characteristics of cannabis impairment. Accident Analysis and Prevention, 92, 219-229. https://doi.org/10.1016/i.aap.2016.04.012

Health Canada. (2017). Canadian Tobacco Alcohol and Drugs Survey (CTADS): 2015 Summary. https://www.canada.ca/en/healthcanada/services/canadian-tobacco-alcohol-drugs-survey/2015summary.html

Health Canada. (2018). Information for health care professionals: Cannabis (marihuana, marijuana) and the cannabinoids. https:// www.canada.ca/en/health-canada/services/drugs-medication/ cannabis/information-medical-practitioners/information-health-careprofessionals-cannabis-cannabinoids.html

Health Canada. (2020). Cannabis for medical purposes under the Cannabis Act: Information and improvements. https://www.canada.ca/ en/health-canada/services/drugs-medication/cannabis/medicaluse-cannabis.html

Heishman, S. J., Singleton, E. G., \& Crouch, D. J. (1996). Laboratory validation study of drug evaluation and classification program: Ethanol, cocaine, and marijuana. Journal of Analytical Toxicology, 20(6), 468-483.

Hill, K. P. (2015). Medical marijuana for treatment of chronic pain and other medical and psychiatric problems: A clinical review. Journal of the American Medical Association, 313(24), 2474-2483. https:// doi.org/10.1001/jama.2015.6199

Huestis, M.A., Barnes, A., \& Smith, M.L. (2005). Estimating the time of last cannabis use from plasma delta-9-tetrahydrocannabinol and 11-nor-9-carboxy-delta9-tetrahydrocannabinol concentrations. Clinical Chemistry, 51 (12), 2289-2295. https://doi.org/10.1373/ clinchem.2005.056838

International Drug Evaluation and Classification Program. (n.d.). Drug Recognition Experts (DREs). https://www.theiacp.org/drug-recognitionexperts-dres

Kibby, T., \& Halcomb, S. E. (2013). Toxicology observation: Nystagmus after marijuana use. Journal of Forensic and Legal Medicine, 20(4), 345-346.

National Conference of State Legislatures. (2021, March 1). State Medical Marijuana Laws. https://www.ncsl.org/research/health/statemedical-marijuana-laws.aspx

National Institute on Drug Abuse (2020, June 11). What are the short-erm effects of cocaine use? https://www.drugabuse.gov/publications/ research-reports/cocaine/what-are-short-term-effects-cocaine-use

Peng, Y. W., Desapriya, E., Chan, H., \& Brubacher, J. R. (2020). Residual blood THC levels in frequent cannabis users after over four hours of abstinence: A systematic review. Drug and Alcohol Dependence, 216, 108177

Porath-Waller, A. J., Beirness, J., \& Beasley, E. E. (2009). Toward a more parsimonious approach to drug recognition expert evaluations. Traffic Injury Prevention 10(6), 513-518.

Porath-Waller, A. J., \& Beirness, D. J. (2010). Simplifying the process for identifying drug combinations by Drug Recognition Experts. Traffic Injury Prevention, 17151, 453-459. https://doi.org/10.1080/153 89588.2010.489199

Ramaekers, J. G., van Wel, J. H., Spronk, D. B., Toennes, S. W., Kuypers, P. C., Theunissen, E. L., \& Verkes, R. J. (2016). Acute drug impairment as a function of cannabis use history. Scientific Reports, 6, 26843, https://doi.org/10.1038/srep26843

Richman, J. E., Golden McAndrew, K., Decker, D., \& Mullaney, S. C. (2004). An evaluation of pupil size standards used by police officers for detecting drug impairment. Journal of the American Optometric Association, 75(3), 175-182. https://doi.org/10.1016/s1529$1839(04) 70037-8$

Richman, J. E. (2017). Drug abuse in your patients. The application of common clinical visual tests in identifying possible drug abuse. Advances in Ophthalmology and Optometry, 2(1), 15-41. https:// doi.org/10.1016/i.yaoo.2017.03.002

Romano, F., Tarnutzer, A. A., Straumann, D., Ramat, S., \& Bertolini, G. (2017). Gaze-evoked nystagmus induced by alcohol intoxication. The Journal of Physiology, 595(6), 2161-2173.

Royal Canadian Mounted Police. (2018a, October 15). Drug recognition expert evaluations. http://www.rcmp-grc.gc.ca/tss-sr/dree-eert-eng.htm

Royal Canadian Mounted Police. (2018b, October 17). Drug-impaired driving. https://www.rcmp-grc.gc.ca/en/drug-impaired-driving

Smith, J., Hayes, C. E., Yolton, R. L., Rutledge, D. A., \& Citek, K. (2002). Drug recognition expert evaluations made using limited data. Forensic Science International, 130(2-3), 167-173. https://doi. org/10.1016/S0379-0738/02/00384-5.

Theunissen, E. L., Kauert, G. F., Toennes, S. W., Moeller, M. R., Sambeth, A., Blanchard, M. M., \& Ramaekers, J. G. (2012). Neurophysiological functioning of occasional and heavy cannabis users during THC intoxication. Psychopharmacology, 220(2), 341-250. https://doi. org/10.1007/s00213-011-2479-x

Yeakel, J. K., \& Logan, B. K. (2013). Blood synthetic cannabinoid concentrations in cases of suspected impaired driving. Journal of Analytical Toxicology, 37(8), 547-551. https://doi.org/10.1093/jat/bkł065 